\title{
EDITORIAL
}

\section{SERUM TESTS FOR SYPHILIS IN EXTREMIS}

This issue contains a paper on "The Interpretation of Serum Reactions in Late Syphilis" recently read to the Medical Society for the Study of Venereal Diseases by Dr. C. P. Heywood, of Bradford, who, perhaps to stimulate discussion, made some provocative statements. During the lively debate which followed, he withdrew slightly from his original ground, as the report of the discussion shows, but the main contentions in his paper are substantially unaffected by his debating retreat.

Heywood has this in common with the multitudes from whom he differs-he admits our fundamentally vague knowledge of the bases of the sero-diagnosis of syphilis. A crude or purified lipoid-containing extract of ox-heart is used in serum tests for syphilis (S.T.S.) and in certain conditions will give a high percentage of " positive" results in cases of known syphilis and of "negative" results in non-syphilitic cases. It is most important to the argument that with this type of test 100 per cent. sensitivity for syphilitic sera has not so far been obtained without an increase in sensitivity to non-syphilitic sera, i.e. a higher percentage of "positive" tests with sera from non-syphilitic cases.

Now Heywood (p. 3) does not claim 100 per cent. sensitivity or 100 per cent. specificity-therefore he implies that some sera from known syphilitic cases give negative reactions and also that some sera from known non-syphilitic cases give positive reactions. He therefore admits that there are certain "acute and chronic diseases known temporarily to produce a false positive S.T.S." $\mathrm{He}$ concludes that

with appropriate safeguards to exclude temporarily false positive reactions, a positive reaction means that the patient has been infected with syphilis at some time in his life,

and that

the concept of the so-called biological false positive reactions to S.T.S. . . . belongs to the realm of armchair metaphysics and has no practical importance in the absence of any readily available method of distinguishing between the patient with the hypothetical biological false positive reaction and the patient with latent syphilis.

What is a " temporarily false positive reaction"? Mohr, Moore, Nelson, and Hill (1950) classify two groups of biological false positive reactors (which many would prefer to call non-syphilitic reactors). The first group of " acute reactors" includes those patients who have suffered from vaccinia, malaria, and atypical virus pneumonia (admitted to provoke positive S.T.S.), and in these the duration of the positive reactions may be from 3 to 6 months. The second group of chronic reactors " consists of a less well-defined set of cases whose S.T.S. usually show low titres and remain positive for more than 6 months and even for years ". The authors, using the treponemal immobilization test (T.P.I.) described by Nelson and Mayer (1949), investigated a group of 256 patients drawn from private practice-126 cases of late treated syphilis, 67 cases of probable latent syphilis, and 63 cases of probable false positive reactors. The T.P.I. test was performed on all ; and of the 126 cases of late treated syphilis 96.8 per cent. were positive, of the 67 cases of probable latent syphilis 76.3 per cent. were positive, and of the 63 cases of probable false positive reactors $11 \cdot 1$ per cent. were positive. It need hardly be pointed out that the clinical assessments were done by two of the authors in ignorance of the results of the T.P.I. test, and that the tests were carried out by the other two authors in ignorance of the nature of the cases the sera came from.

This seems to provide, so far as it goes, adequate evidence that a means does exist of distinguishing between " true syphilitic" and "non-syphilitic" reactors, though it should be noted that, as in most biological phenomena, 100 per cent. agreement was not obtained.

Kahn (1950a), discussing non-syphilitic reactors, draws attention to the phenomenon of lipoid antigens producing precipitation (positive reactions) with normal sera, and demonstrates that by variations, in e.g. the concentration of saline used in the 
test or in the duration and temperature of incubation, most sera can be made to give precipitation with the lipoid antigen normally used in S.T.S.

It is worth while to quote Kahn (1950b) :

It would be more correct to emphasize that : (1) any and all diseases and any and all injections of a protein or organic nature whether they be for immunizations or other reasons may in some individuals call forth positive reactions with tests for syphilis ; (2) in isolated instances, such reactions occur in normal individuals, assuciated perhaps with various factors including diet ; (3) the reactions are generally temporary lasting for some weeks to several months, but they may exist for years.

Before Heywood's pessimistic conclusion, that with few exceptions all patients showing positive
S.T.S. are syphilitic, is accepted as a guide for practice, venereologists will in most cases, we think, choose to insist that such a promising and apparently discriminating test as the treponemal immobilization test should become more readily available, and will thus avoid not only the professional crime of treatment without diagnosis but also much misery and unhappiness to their patients.

\section{REFERENCES}

Kahn, R. L. (1950a). " Serology with Lipid Antigen". Baillière, Tindall and Cox, London. (1950b). Ibid, p. 190.

Mohr, C. F., Moore, J. E., Nelson, R. A., and Hill, J. H. (1950). Amer. J. Syph., 34, 405.

Nelson, R. A., and Mayer, M. M. (1949). J. exp. Med., 89, 369. 